\title{
A Preliminary Strategy for Fall Prevention in the ASBGo Smart Walker*
}

\author{
Ana Pereira $^{1}$, Nuno Ferrete Ribeiro ${ }^{1}$, Cristina P. Santos ${ }^{1}$
}

\begin{abstract}
Fall-related injuries affect a large part of the population and related costs. Thus, there is a concern in studying a fall prevention strategy to minimize the consequences of falls. Walkers are assistive devices used to improve the balance, stability and reduce the load on the lower limb of the user. In this sense, there is a concern to improve the safety in smart walkers and, consequently, to prevent falls in these devices. However, in this field, the only approach is to stop the walker in risk situations. So, the aim of this paper is to define a preliminary strategy to prevent a fall event in the Adaptive System Behaviour Group (ASBGo) Smart Walker. For ASBGo Smart Walker, two modes of security are discussed in this paper. One approach is based on monitoring the center of mass and changing the trajectory when a near fall is detected. The other mechanism consists only in to stop the walker when a dangerous situation is detected. The first or the second mode are activated depending if the user drives the walker with the forearm on forearm support or not.
\end{abstract}

\section{INTRODUCTION}

According to World Health Organization (WHO), 646 thousand people died around the world as a result of a fall. The WHO defines a fall as "an event which results in a person coming to rest inadvertently on the ground or floor or other lower level" [1]. Falls do not always happen in the same way and in the same direction. There are different types of falls, such as falling forward, sideward, backward [2], from the bed and from the chair [3]. Regarding phases of falls, different phases are considered by different authors. For instance, Hsieh et al. [4] divide the fall event into three phases: free fall phase, impact phase, and rest phase. The free fall phase is characterized by abrupt movements. The impact phase consists the person shock on the ground, and lately, in the rest phase the person remains immobilized.

Falls have a high impact on peoples lives. There are many injuries associated with falling, for example, dislocations, laceration, fractures, and hematomas. The fear of falling and depression are also noted as fall-related effects [5], [6]. Other consequences, such as dehydration and hypothermia are related to falls due to inability to stand up [5].

\footnotetext{
*This work has been supported by the FCT - Fundação para a Ciência e Tecnologia - with the scholarship reference PD/BD/141515/2018, by the FEDER funds through the COMPETE 2020 - Programa Operacional Competitividade e Internacionalização (POCI) and P2020 with the Reference Project EML under Grant POCI-01-0247-FEDER-033067, and through the COMPETE 2020 - POCI - with the Reference Project under Grant POCI01-0145-FEDER-006941.

${ }^{1}$ A. Pereira, N. F. Ribeiro and C. P. Santos are with the Center for MicroEletroMechanical Systems (CMEMS), University of Minho, 4800-058, Guimarães, Portugal ana.rita.23@hotmail.com, nuno.ferrete.ribeiro@gmail.com, cristinaddei.uminho.pt
}

There are several risk factors that increase the likelihood of falling. The risk factors can be related to the patients, for instance, age, mental confusion, poor balance, dizziness and dementia [7]. Nevertheless, the risks of falling are also related to the environment [7], [8], such as tripping, inappropriate footwear and poor lighting.

Falls have a high cost and represent a burden to society. In 2012 , the direct costs of non-fatal falls were $\$ 616.5$ million, and in relation to fatal falls, the total cost was $\$ 30.3$ billion. In 2015 , the cost increase to $\$ 637.2$ million and $\$ 31.3$ billion to non-fatal and fatal falls, respectively [9].

In order to minimize the fall-related impact, a few systems and strategies have been developed. The approaches are based on non-wearable and wearable sensors. Different nonwearable sensors are used to detect falls, such as depth cameras [10], thermal sensors [11] and ultrasonic array [12]. Regarding wearable sensors, inertial measurement units (IMUs) [13] and force sensing resistors [14] are well-known examples. To prevent the user from falling, i.e., the user did not fall to the ground, a few sensors are used, e.g. electromyography probes [15], IMUs, cameras and reflective markers [16].

There is a concern to detect or prevent fall with smart medical assistive devices, such as smart walkers [17]-[24]. The smart walkers can be equipped with several sensors which allow extracting different user-related features. Thus, information about the user's gait can be achieved. All the information acquired can be used to improve the user's safety and help predict a fall event in this type of assistive devices. Different approaches have been developed in order to prevent the user from fall. However, the strategies implemented in smart walkers to prevent a fall consist of stopping the walker when a near fall is detected. So, the aim of this article is to propose a different approach to prevent a fall event using the Adaptive System Behaviour Group (ASBGo) Smart Walker.

The rest of the paper is organized as follows. In section II, the sensors and the functionalities of the ASBGo Smart Walker are described. In section III, an overview of the security modes implemented in smart walkers found it in literature is presented. In section IV, it is discussed the strategy under development on ASBGo Smart Walker. Finally, section $\mathrm{V}$ contains the conclusion.

\section{ASBGo SMART WALKer}

The Adaptive System Behaviour Group (ASBGo) Smart Walker was developed by the Adaptive System Behaviour Group. This assistive device was created for rehabilitation and physical therapy in order to aid patients with gait 
disorders and improve their physical conditions. The ASBGo Smart Walker provides an alternative to standard walkers and a safe and quality rehabilitation.

The Smart Walker device is equipped with several sensors, as shown in Fig. 1. The sensors located at the upper and middle part of walker allow the extracting of different patient-related characteristics [25]-[29]. The bottom part of the Smart Walker contains sensors to detect obstacles during its use [30]. The acquired patients' information is about their stability, balance, gait, and posture. A few functionalities were developed with this information, in order to improve patient's rehabilitation [25].

Different modes of operation can be chosen when patients use the Smart Walker, such as local, remote and autonomous driving, and treadmill. These modes of operation allow the physiotherapist to choose the most appropriate mode depending on the patient's difficulties. In the local mode, the user can choose the parameters of driving, such as the velocity and the curvature of the walker. Here, the user can guide the walker. Usually, this mode is used when the patient is in the advanced stage of recovery and, consequently, has more mobility. On the other hand, in remote driving, the physiotherapist has a joystick and controls the walker's movement in the environment. A treadmill mode can also be chosen. In this mode, the user while uses the treadmill can benefit the walker functionalities at the same time. Finally, the autonomous mode allows the physiotherapist or the user to choose the desired coordinate position.

It is important to emphasize that the patient can use the walker in two different ways: i) with the forearm on forearm support driving the walker through the handlebar (close to touching monitor) or ii) guide the walker through the handle grip (positioned before the wooden table).

In a previous study, a strategy to prevent falls has already been addressed. If the distance between the walker and the user decreases abruptly, it means that a near fall can occur and the walker stops. In the case of the user does not have

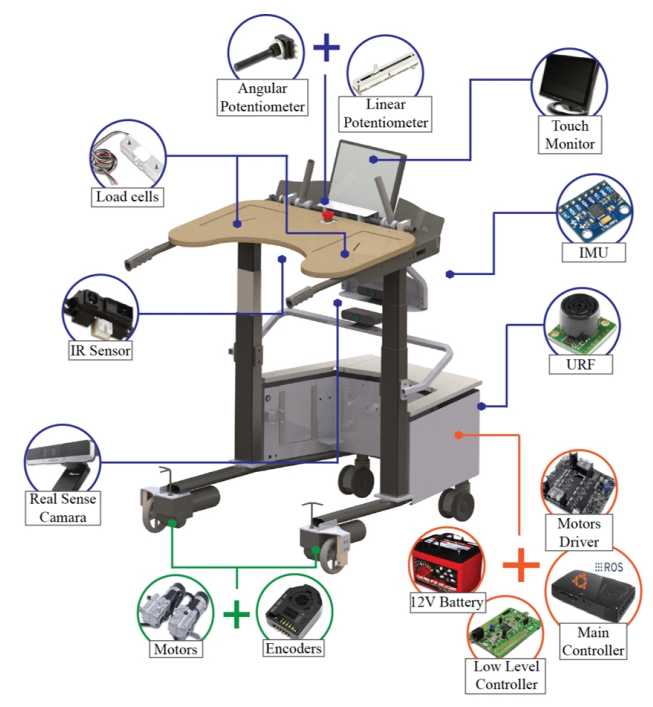

Fig. 1: The architecture and sensors' location of the ASBGo Smart Walker. the forearm on forearm support the walker also stops. Two force sensors have also been positioned in handlebar in order to know if the user has the hands on the handlebar. Again, if the user does not have his hands on handlebar the walker stops. The walker does not move backward, even if the user pushes it [31].

\section{SAFETY MODE}

A preliminary study was performed with the objective of understanding which are the most relevant features to prevent a fall event in smart walkers. Through this analysis was possible to define the approach to implement in ASBGo Smart Walker.

Based on the literature, it was possible to conclude that there is a concern related to the users' safety when using an assistive device. Concerning smart walkers, several sensors were used to implement and improve the safety mode of these devices. Force sensors, laser range finder (LRF), cameras, as well as IMUs contribute to the safety mode of the smart walker [17]-[24]. It means that not only non-wearable sensors but also wearable sensors were used in order to prevent the user from fall.

The distance between the user and the walker, the center of gravity or center of pressure, and the force exerted on the handle are important features to prevent a fall event [17]-[24]. Of all the strategies implemented in several smart walkers, the final outcome is always stopping the walker.

\section{Safety Mode ASBGo Smart Walker}

As mentioned in the second section, the ASBGo Smart Walker may be driving with the forearm on the forearm support through the handlebar (Fig.2), or alternatively, with the hands on handle grip.

Fig. 3 shows a schematic of the strategy being developed. The first step is to choose the appropriate mode for the patient. Then, the position of the forearm and the hands during the use of the walker is selected. If the user is not using the handlebar, safe mode 1 is activated, otherwise safe mode 2 is activated. Two modes of security were implemented to achieve a strong strategy. In safe mode 2, the support area is bigger than in safe mode 1 . The user has the forearm supported on the wooden table, while in mode 1 the only contact points of the user to the walker are the hands supported on handle grip.

\section{A. Safe Mode 1}

Based on the literature and the architecture of the ASBGo Smart Walker, a strategy to prevent a fall event has been defined. The approach developed is based on several features related to the patients. These characteristics are the user's center of mass (COM), the correct position of the hands, spatiotemporal parameters of gait, and the direction of the fall event.

According to Taghvaei et al. [19] the COM is an important feature to detect a fall. Thus, the COM has been defined as the main feature to detect a near fall event in this mode. The user's COM will be continuously calculated in real-time 


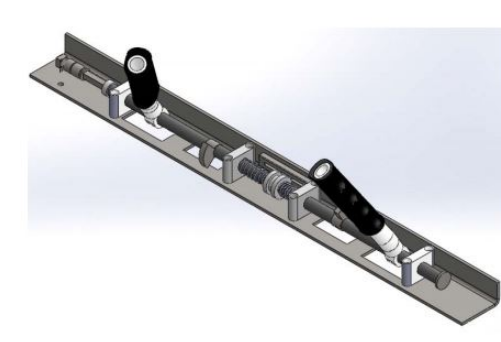

Fig. 2: The handlebar of the Smart Walker [37].

while the user is driving the Smart Walker. The sensor chosen to extract this feature is a camera as used by Taghvaei et al. [19] and the selected frame rate was 30 frames per second based in [32]-[35]. According to Mastorakis et al. [33], a high frame rate in real-time systems is important because the fall occurs in a very short time interval.

The correct position of the hands on the walker is also an important indicator of the dangerous situation. If the hands are not on handle grip, it may indicate that the user has become unbalance and, as a result, may fall. The force sensors on the handle grips as well as the camera are the approaches selected to detect if the user's hands are correctly positioned [27], [31].

The distance between the user and the walker is considered another important feature. If the distance decreases quickly, it is possible that the user is falling forward. On the other hand, if the distance increases abruptly, it is possible that the user is falling backward. In order to calculate the distance an infrared sensing will be used as Martins et al. [31].

It is also intended to extract the spatiotemporal parameters of gait through the LRF [28] and use the features extracted to detect a pre-fall step. It should be noted that no strategy was found in the literature that detects a pre-fall step with a smart walker.

All the features aforementioned will be calculated in order to detect a near fall event. Regarding fall prevention strategies in the literature, the final outcome is always stopping the walker. Those mechanisms in case of sideward fall may not provide an efficient support.

The first step for fall prevention approach is to determine the direction of the fall. Next, the ASBGo Smart Walker must change the trajectory in accordance with the direction of the fall, in order to enable the user to recover the balance quickly. When the walker changes trajectory there may be obstacles in the environment that must be considered. After adjusting the trajectory, the walker stops to provide support. A similar approach was implemented in a robot cane by Pei Di et al. [36]. While the safety mode is not turned off by the user or physiotherapist, the walker remains motionless. When the security mode is disabled, the features are continuously acquired and analyzed to detect a possible fall.

\section{B. Safe Mode 2}

In the case of the user driving the walker with the forearm on the forearm support, safety mode 2 will be activated. With this mode, four features will be extracted to detect a fall event: the distance between the user and the walker,

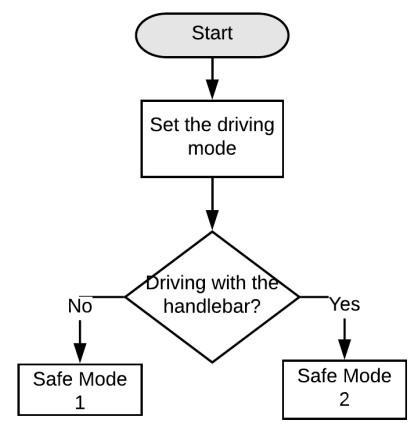

Fig. 3: Scheme of the fall prevention strategy to be implemented in ASBGo Smart Walker.

the gait characteristics, the signal of the load cells on the forearm support and the user's COM. In relation to the first two features mentioned, the functioning is the same as the previous mode. The load cells signals are extracted to verify if the user has the forearm on the forearm support. If the user does not have the forearm correctly positioned, a potentially dangerous situation occurs. Regarding COM in this mode is not possible use the camera. Thus, the chosen sensor was the IMU, which will be placed on the lower back. The sampling rate of $102.4 \mathrm{~Hz}$ was selected according to Doeheny et al. [38].

Concerning the fall prevention strategy in this mode, it consists of stopping the walker when a dangerous situation is detected. This outcome is similar to that found in the literature. The walker will only be operational again when the safety mode is disabled by the technician. In this mode, the change of trajectory is not applied because the user is very close to the walker. Thus, the wooden table already provides a large area of support, whether the user falls forward or to both sides.

\section{Other safety measures}

The ASBGo Smart Walker currently has also implemented one emergency button. In case of a dangerous situation, the physiotherapist can press the button and the walker stops. The fact that the ASBGo Smart Walker does not move backward and avoid obstacles are other safety measures already implemented that improves the user safety.

\section{CONCLUSION}

The preliminary strategy developed for the ASBGo Smart Walker was defined in order to improve the safety of the patient while using the device. The outcomes found in the literature related to the smart walker are based on stopping the walker when a fall event is detected. Thus, the approach presented in this paper is more robust when compared to found in the literature. It is expected that the methodology proposed in mode 1 provides an improvement of user's safety, especially in sideward falls. Regarding future work, it consists in testing the whole proposed system and making this strategy even stronger. Once this Smart Walker is targeted for rehabilitation, it would be interesting to implement a biofeedback system to improve the user balance. 


\section{REFERENCES}

[1] W. H. Organization, "Falls," 2018. [Online]. Available: http://www.who.int/news-room/fact-sheets/detail/falls [Accessed: 17Nov-2018].

[2] N. Noury, A. Fleury, P. Rumeau, A. K. Bourke, G. Ó. Laighin, V. Rialle, and J. E. Lundy, "Fall detection - Principles and methods," in Annual International Conference of the IEEE Engineering in Medicine and Biology - Proceedings, 2007, pp. 1663-1666.

[3] Xinguo Yu, "Approaches and principles of fall detection for elderly and patient," in HealthCom 2008 - 10th International Conference on e-health Networking, Applications and Services. IEEE, Jul 2008, pp. $42-47$.

[4] C.-Y. Hsieh, K.-C. Liu, C.-N. Huang, W.-C. Chu, and C.-T. Chan, "Novel Hierarchical Fall Detection Algorithm Using a Multiphase Fall Model," Sensors (Basel, Switzerland), vol. 17, no. 2, Feb 2017.

[5] K. Obrant, in Management of Fractures in Severely Osteoporotic Bone, 1st ed., K. Obrant, Ed. London: Springer London, 2000, ch. IV, pp. 333-336.

[6] M. Terroso, N. Rosa, A. Torres Marques, and R. Simoes, "Physical consequences of falls in the elderly: A literature review from 1995 to 2010," pp. 51-59, Apr 2014.

[7] M. Boltz, "Fall Prevention: Assessment, Diagnoses, and Intervention Strategies," in Evidence-Based Geriatric Nursing Protocols for Best Practice, 4th ed., M. Boltz, E. Capezuti, T. Fulmer, and D. Zwicker, Eds. New York: Springer Publishing Company, 2012, ch. 15, pp. 273-276.

[8] T. Cimilli Ozturk, R. Ak, E. Unal Akoglu, O. Onur, S. Eroglu, and M. Saritemur, "Factors Associated With Multiple Falls Among Elderly Patients Admitted to Emergency Department," International Journal of Gerontology, vol. 11, no. 2, pp. 85-89, 2017.

[9] E. R. Burns, J. A. Stevens, and R. Lee, "The direct costs of fatal and non-fatal falls among older adults United States," Journal of Safety Research, vol. 58, pp. 99-103, 2016.

[10] X. Kong, L. Meng, and H. Tomiyama, "Fall detection for elderly persons using a depth camera," in 2017 International Conference on Advanced Mechatronic Systems (ICAMechS). IEEE, Dec 2017, pp. 269-273.

[11] C. Taramasco, T. Rodenas, F. Martinez, P. Fuentes, R. Munoz, R. Olivares, V. H. C. De Albuquerque, and J. Demongeot, "A Novel Monitoring System for Fall Detection in Older People," IEEE Access, vol. 6, pp. 43 563-43 574, 2018.

[12] C. Nadee and K. Chamnongthai, "Ultrasonic array sensors for monitoring of human fall detection," in 2015 12th International Conference on Electrical Engineering/Electronics, Computer, Telecommunications and Information Technology (ECTI-CON). IEEE, jun 2015, pp. 1-4.

[13] T. de Quadros, A. E. Lazzaretti, and F. K. Schneider, "A Movement Decomposition and Machine Learning-Based Fall Detection System Using Wrist Wearable Device," IEEE Sensors Journal, vol. 18, no. 12, pp. 5082-5089, Jun 2018.

[14] Y. Tao, H. Qian, M. Chen, X. Shi, and Y. Xu, "A Real-time intelligent shoe system for fall detection," in 2011 IEEE International Conference on Robotics and Biomimetics. IEEE, Dec 2011, pp. 2253-2258.

[15] G. Rescio, A. Leone, and P. Siciliano, "Supervised machine learning scheme for electromyography-based pre-fall detection system," Expert Systems with Applications, vol. 100, pp. 95-105, Jun 2018.

[16] Jian Liu and T. E. Lockhart, "Development and Evaluation of a Priorto-Impact Fall Event Detection Algorithm," IEEE Transactions on Biomedical Engineering, vol. 61, no. 7, pp. 2135-2140, Jul 2014.

[17] Y. Hirata, S. Komatsuda, and K. Kosuge, "Fall prevention control of passive intelligent walker based on human model," 2008 IEEE/RSJ International Conference on Intelligent Robots and Systems, IROS, pp. 1222-1228, 2008.

[18] S. Taghvaei, Y. Hirata, and K. Kosuge, "Vision-based human state estimation to control an intelligent passive walker," in 2010 IEEE/SICE International Symposium on System Integration. IEEE, Dec 2010, pp. $146-151$.

[19] S. Taghvaei and K. Kosuge, "Image-based fall detection and classification of a user with a walking support system," Frontiers of Mechanical Engineering, vol. 13, no. 3, pp. 427-441, 2018.

[20] W. Xu, J. Huang, and L. Cheng, "A Novel Coordinated Motion FusionBased Walking-Aid Robot System," Sensors, vol. 18, no. 9, p. 2761, Aug 2018.

[21] S. Irgenfried and H. Wörn, "Motion Control and Fall Prevention for an Active Walker Mobility Aid," in Mechanisms and Machine Science, ser. Mechanisms and Machine Science, V. Petuya, C. Pinto, and E.-C. Lovasz, Eds. Dordrecht: Springer Netherlands, 2014, pp. 157-164.

[22] J. Huang, W. Xu, S. Mohammed, and Z. Shu, "Posture estimation and human support using wearable sensors and walking-aid robot," Robotics and Autonomous Systems, vol. 73, pp. 24-43, Nov 2015.

[23] W.-H. Mou, M.-F. Chang, C.-K. Liao, Y.-H. Hsu, S.-H. Tseng, and L.C. Fu, "Context-aware assisted interactive robotic walker for Parkinson's disease patients," in 2012 IEEE/RSJ International Conference on Intelligent Robots and Systems. IEEE, Oct 2012, pp. 329-334.

[24] M. Azqueta-Gavaldon, I. Azqueta-Gavaldon, M. Woiczinski, K. Bötzel, and E. Kraft, "Automatic Braking System and Fall Detection Mechanism for Rollators," in Proceedings of the 6th International Conference on Bioinformatics and Biomedical Science - ICBBS '17. New York, New York, USA: ACM Press, 2017, pp. $158-161$.

[25] A. Tereso, M. Martins, C. P. Santos, M. Vieira da Silva, L. Gonçalves, and L. Rocha, "Detection of Gait Events and Assessment of Fall Risk Using Accelerometers in Assisted Gait," in 2014 11th International Conference on Informatics in Control, Automation and Robotics (ICINCO), 2014, pp. 788-793.

[26] S. Page, M. M. Martins, L. Saint-Bauzel, C. P. Santos, and V. Pasqui, "Fast embedded feet pose estimation based on a depth camera for smart walker," in 2015 IEEE International Conference on Robotics and Automation (ICRA). IEEE, May 2015, pp. 4224-4229.

[27] I. Caetano, J. Alves, J. Goncalves, M. Martins, and C. P. Santos, "Development of a Biofeedback Approach Using Body Tracking with Active Depth Sensor in ASBGo Smart Walker," in 2016 International Conference on Autonomous Robot Systems and Competitions (ICARSC). IEEE, May 2016, pp. 241-246.

[28] M. Martins, A. Frizera, R. Ceres, and C. Santos, "Legs tracking for walker-rehabilitation purposes," in 5th IEEE RAS/EMBS International Conference on Biomedical Robotics and Biomechatronics. IEEE, Aug 2014, pp. 387-392.

[29] M. Martins, C. Santos, E. Seabra, L. Basilio, and A. Frizera, "A new integrated device to read user intentions when walking with a Smart Walker," in 2013 11th IEEE International Conference on Industrial Informatics (INDIN). IEEE, Jul 2013, pp. 299-304.

[30] V. Faria, J. Silva, M. Martins, and C. Santos, "Dynamical system approach for obstacle avoidance in a Smart Walker device," in 2014 IEEE International Conference on Autonomous Robot Systems and Competitions (ICARSC). IEEE, May 2014, pp. 261-266.

[31] M. Martins, C. Santos, and A. Frizera, "Online control of a mobility assistance Smart Walker," in 2012 IEEE 2nd Portuguese Meeting in Bioengineering (ENBENG). IEEE, Feb 2012, pp. 1-6.

[32] S. Gasparrini, E. Cippitelli, S. Spinsante, and E. Gambi, "A Depth-

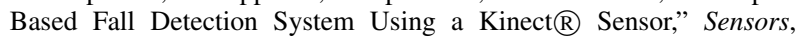
vol. 14, no. 2, pp. 2756-2775, Feb 2014.

[33] G. Mastorakis and D. Makris, "Fall detection system using Kinect's infrared sensor," Journal of Real-Time Image Processing, vol. 9, no. 4, pp. 635-646, Dec 2014.

[34] C. Kawatsu, J. Li, and C. J. Chung, "Development of a Fall Detection System with Microsoft Kinect," in Advances in Intelligent Systems and Computing, 2013, pp. 623-630.

[35] L. Panahi and V. Ghods, "Human fall detection using machine vision techniques on RGBD images," Biomedical Signal Processing and Control, vol. 44, pp. 146-153, Jul 2018.

[36] P. Di, J. Huang, S. Nakagawa, K. Sekiyama, and T. Fukuda, "Fall detection and prevention in the elderly based on the ZMP stability control," in 2013 IEEE Workshop on Advanced Robotics and its Social Impacts. IEEE, Nov 2013, pp. 82-87.

[37] J. Alves, E. Seabra, I. Caetano, J. Goncalves, J. Serra, M. Martins, and C. P. Santos, "Considerations and Mechanical Modifications on a Smart Walker," in 2016 International Conference on Autonomous Robot Systems and Competitions (ICARSC). IEEE, May 2016, pp. 247-252.

[38] E. P. Doheny, D. McGrath, B. R. Greene, L. Walsh, D. McKeown, C. Cunningham, L. Crosby, R. A. Kenny, and B. Caulfield, "Displacement of centre of mass during quiet standing assessed using accelerometry in older fallers and non-fallers," in 2012 Annual International Conference of the IEEE Engineering in Medicine and Biology Society. IEEE, Aug 2012, pp. 3300-3303. 